\title{
La Última Milla: Los desafíos éticos de la pobreza extrema y la vulnerabilidad en la Agenda 2030 para el Desarrollo Sostenible de Naciones Unidas
}

\author{
MARTA PEDRAJAS* \\ Programa de las Naciones Unidas para el Desarrollo (España) \\ marta.pedrajas@undp.org
}

\begin{abstract}
Resumen
Se analiza desde el punto de vista ético la acción internacional en desarrollo, a través del acuerdo de la Asamblea General de Naciones Unidas: la Agenda 2030 para el Desarrollo Sostenible. Los desafíos éticos de la nueva agenda son numerosos, pero hay un compromiso ético de primer orden: no dejar a nadie atrás. Para ejercitar esta acción, el PNUD y otros organismos como el Banco Mundial o UNICEF están trabajando con enfoques multidimensionales. Se trata de cubrir lo que se conoce como la Última Milla, los casi mil millones de personas que siguen viviendo en pobreza extrema, y que están excluidos de toda acción de desarrollo. En este artículo se dan las pautas de cómo se debe abordar desde la multidimensionalidad esta pobreza extrema y la vulnerabilidad y cumplir el mandato de la Agenda 2030, no dejar a nadie atrás.
\end{abstract}

Palabras clave: Desarrollo Humano, pobreza, Agenda 2030, Naciones Unidas, última milla.

\section{The Last Mile: The Ethical Challenges of Extreme Poverty and Vulnerability in the UN 2030 Agenda for Sustainable Development}

\begin{abstract}
The international action under development is analyzed from the ethical point of view, through the agreement of the General Assembly of the United Nations: the Agenda 2030 for Sustainable Development. The ethical challenges of the new agenda are numerous, but there is an ethical commitment of the first order: do not leave anyone bebind. To implement this action, UNDP and other agencies such as the World Bank or UNICEF are working with multidimensional approaches. It is about covering what is known as the Last Mile, the almost one billion people who continue to live in extreme poverty, and who are excluded from all development action. This article gives guidelines on how to tackle this extreme poverty and vulnerability and to fulfill the mandate of the Agenda 2030 from the multidimensionality, leaving no one bebind.
\end{abstract}

Key words: Human Development, poverty, 2030 Agenda, United Nations, last mile.

* Doctora en Filosofía y Licenciada en Económicas. Especialista en Políticas de Desarrollo del Programa de Naciones Unidas para el Desarrollo (PNUD). Entre sus publicaciones cabe mencionar Getting to the Last Mile in Least Developed Countries (2016, junto con S. Choritz).

Las opiniones de este articulo son de la autora y no representan necesariamente la opinión del Secretariado de Naciones Unidas, del Programa de Naciones Unidas para el Desarrollo o ninguna de las organizaciones afiliadas ni de sus Estados Miembros. 


\section{INTRODUCCIÓN: ÉTICA GLOBAL PARA UN MUNDO GLOBAL}

La dignidad de personas, el cuidado de planeta, la generación de prosperidad y el logro de la paz a través de las alianzas entre todos, marcan los principios de la nueva Agenda 2030 para el Desarrollo Sostenible, aprobada en Naciones Unidas en septiembre de 2015 por todos países del mundo (Naciones Unidas, 2015a).

Una agenda que contiene los principios inspirados en la Carta de $\mathrm{Na}$ ciones Unidas (1945) y una visión para el mundo en 2030 que se concreta en los 17 Objetivos de Desarrollo Sostenible (ODS), con sus metas e indicadores (Naciones Unidas, 2016a), los medios de implementación y el sistema de seguimiento y revisión para impulsar y garantizar su cumplimiento en todos los países y regiones. Una agenda que además se ha visto reforzada por otros acuerdos internacionales de los dos últimos años, entre los que destacan especialmente las grandes cumbres de desarrollo del mismo año 2015: el acuerdo de Financiación del Desarrollo establecido en la Agenda de Acción de Addis Abeba (Naciones Unidas, 2015b) y el Acuerdo de Paris contra el Cambio Climático (United Nations Framework Convention on Climate Change, 2015).

La Agenda 2030 marca el rumbo, la ruta a seguir para los próximos quince años. Y esta hoja de ruta está construida sobre los progresos en términos de desarrollo de las últimas décadas (PNUD, 2017), pero abordando desde un enfoque global, donde el progreso convive con una pobreza persistente y extrema para casi mil millones de personas, un aumento de las desigualdades y una degradación del planeta y del cambio climático que nos desafía cada día.

Pero también hay datos y evidencias para la esperanza, que nos llevan a afirmar con el Informe de Desarrollo Humano que "algo que en su momento se consideraba impensable, ahora podemos decir que se ha vuelto imparable" (PNUD, 2017: 4). Efectivamente, el mundo ha experimentado un gran avance en términos de desarrollo en los últimos veinticinco años, los Informes de Desarrollo Humano del PNUD o el seguimiento de la Agenda de los Objetivos de Desarrollo del Milenio (ODM) nos ha dado buena cuenta de ello (United Nations, 2005-2015). Las medias de desarrollo humano han aumentado para todos los países y regiones del mundo. El mayor logro de las dos últimas décadas, en la era de los ODM, ha sido sin duda la reducción de la pobreza extrema. Era el Objetivo número 1 en el año 2000 y se consiguió en 2010, según el Banco Mundial. Más de mil millones de personas han escapado de la pobreza, reduciéndose de un 35\% a un 11\% (Banco Mundial, 2017). La tasa de mortalidad infantil se redujo 
en más de la mitad, siendo el descenso más marcado en África Subsahariana (Naciones Unidas, 2016b; PNUD, 2017: 26). Así mismo, 2.100 millones de personas obtuvieron acceso a servicios de saneamiento mejorados y 2.600 millones a una fuente de agua potable mejorada (Naciones Unidas, 2015b). Y podríamos dar muchos más resultados esperanzadores de desarrollo.

Pero las medias y los logros no pueden ocultar a los que se han quedado fuera del progreso. Hoy día, según el Banco Mundial todavía hay casi mil millones de personas (766 millones, de los que 385 millones son niños), que se siguen quedando atrás, excluidas del progreso y de los frutos del desarrollo (Banco Mundial, 2017; UNICEF \& World Bank Group, 2016). Es el Bottom Billion de Paul Collier (2007), the Last Mile del desarrollo, la pobreza extrema persistente, reforzada por la desigualdad y la exclusión social (Pedrajas \& Choritz, 2016; Chandy, Kato \& Kharas, 2015). La Última Milla no es un concepto de espacio físico, ni se refiere solo a la falta de recursos. Es mucho más complejo y profundo, es una falta de derechos fundamentales, y de oportunidades, una combinación de exclusión y desigualdad que refuerza la pobreza extrema. Es una última milla moral, probablemente la más difícil de cubrir para los programas de desarrollo, pero también la más necesaria, es lo que falta para erradicar la pobreza extrema del planeta.

Para abordar esta complejidad, también es necesario tener en cuenta algunas características del contexto en que nos encontramos, además de esta pobreza extrema persistente y latente y de los desafíos crecientes del cambio climático, en los últimos años estamos asistiendo a un agravamiento de los conflictos y un aumento de las crisis humanitarias de alto nivel (Martínez-Solimán \& Conceiçāo, 2017): la crisis del Ébola, Siria, Yemen, República Centroafricana, Sudán del Sur... tragedias en sí mismas y que, en un mundo global, extienden sus efectos a todos los países. La huida de la miseria, de los conflictos, el miedo, el terrorismo o los efectos del cambio climático... ha provocado un aumento del desplazamiento de población de manera interna en muchos países y de refugiados hacia el exterior que está sobrepasando todas las previsiones y cuestionando los sistemas de acogida. Ser testigos diarios del desplazamiento de familias enteras, niños, adultos, jóvenes, transitando por vías muy inseguras, cuando no letales, hasta llegar en condiciones indignas a las costas europeas es algo que nos cuestiona desde el punto de vista político, económico, social y también desde un punto de vista ético, desde los propios valores del desarrollo humano. Es la realidad de la Globalización de la Indiferencia, que ha denunciado tantas veces el Papa Francisco (2015).

Problemas globales requieren respuestas globales. Y es cierto que la comunidad internacional lleva muchas décadas afrontando los procesos 
del desarrollo y del subdesarrollo, del conflicto y, más recientemente del cambio climático. Una respuesta que debe ser política, económica, social, medioambiental y ética, en definitiva, una respuesta de desarrollo humano y sostenible. En este artículo argumentamos que se debe incluir también una reflexión ética desde sus fundamentos, desde sus principios y valores, y tener en cuenta las consecuencias en su aplicación.

La reflexión que vamos a hacer en este artículo se nutre desde la ética del desarrollo humano de Amartya Sen enriquecida por otros autores, y que dialoga también con los principios éticos de otras tradiciones. Desde esta ética del desarrollo humano vamos a analizar los principales desafíos de la Agenda 2030 para el desarrollo sostenible, centrándonos en el significado y alcance de su compromiso ético más fuerte: no dejar a nadie atrás.

\section{LOS PRINCIPIOS DE LA ÉTICA DEL DESARROLLO HUMANO}

Pero antes de entrar en la agenda 2030 vamos a recordar, brevemente, los fundamentos en los que basamos la ética del desarrollo, y en concreto, la ética del desarrollo humano (Pedrajas, 2005; 2007). Se trata de un concepto fruto de una larga trayectoria de pensamiento económico y ético en torno al tema del desarrollo. Efectivamente, la filosofía lleva muchos años y una larga trayectoria apostando por una recuperación ética de los planteamientos políticos y económicos que den pie a una sociedad justa, en occidente y en oriente, desde Aristóteles a Kautilya, y por supuesto con Adam Smith, tal y como nos recuerda Amartya Sen (1989: 21).

Como muchas éticas aplicadas (ética empresarial, bioética, ética de los medios de comunicación, ética de las profesiones, etc.) también la ética del desarrollo nace a mediados del siglo XX (Cortina, 1993). En este caso, de la mano de Denis Goulet, pofesor de Economía y de Educación para la Justicia de la University of Notre Dame, y autor de obras tan relevantes como The Cruel Choice: A New Concept in the Theory of Development (Goulet, 1985), que refleja el drama humano de tener que elegir entre el esperado bienestar futuro a costa de mantener el sufrimiento de la generación presente, algo que grosso modo proponían las teorías del desarrollo basadas en la inversión en las capas productivas y en la industrialización de mediados del siglo XX (Pedrajas, 2005). Su obra más importante para nosotros es Ética del desarrollo (Goulet, 1999).

Esta tradición se desarrolla con otros autores, anteriores y posteriores a Goulet (desde Prebisch, Gandhi hasta Streeten), sí es cierto que han sido sus discípulos los que han seguido impulsando la ética del desarrollo, no sólo como reflexión, sino también como organización que goza en la actualidad de una destacada presencia en los distintos foros internacionales. 
Destacan, y muy considerablemente, en este sentido la tradición ética española encabezada por Adela Cortina (2017; también 1997; 2001; 2002), Jesús Conill (2004), Emilio Martínez (2000) o Agustín Domingo (1997). A todos ellos se suma, sin duda alguna, David Crocker, primer presidente de la asociación IDEA (International Development Ethics Association, www.development-ethics.org) creada en 1987 en Costa Rica. Otras asociaciones a tener muy presentes son la Human Development and Capability Association (HDCA, www.capabilityapproach.com), fundada en 2004, que ostenta la presidencia de honor de Amartya Sen. Y también la Fundación ÉTNOR (Fundación para la Ética de los Negocios y las Organizaciones, www.etnor.org) creada en 1994 bajo la dirección de Adela Cortina.

La ética del desarrollo, por tanto, surge al albor de las éticas aplicadas que consisten en una reflexión filosófica sobre los principios, valores y fines que fundamentan y orientan el saber y la acción humana en una disciplina concreta. En este caso, nos preguntamos qué se entiende por desarrollo, cuáles son los valores, principios y fines que lo orientan, cuál es el modelo de persona y la teoría de justicia social que lo fundamenta.

Hay distintas respuestas de ética del desarrollo, pero para nosotros, la ética del desarrollo está basada en el modelo de desarrollo humano, iniciado sobre todo por Amartya Sen (1999; Pedrajas, 2005), que ha tenido una aplicación muy práctica dado que ha inspirado la acción de los últimos 25 años del Programa de Naciones Unidas para el Desarrollo (PNUD, 1990-2017). Amartya Sen, ha sido un pionero dentro de la economía que ha reflexionado sobre la justicia social, sobre el modelo de ser humano y ha impulsado los fundamentos morales de la economía y del desarrollo. Es importante destacar también que en esta labor ha logrado el máximo reconocimiento al obtener el Premio Nobel de Economía en 1998.

Es un modelo ético del desarrollo, que ha ido evolucionando con otros autores, y que basa en tres pilares: desarrollo como libertad, enfoque de las capacidades y autonomía moral (Pedrajas, 2005; 2007). Y su definición clásica es aquella que entiende el desarrollo humano como la expansión de las capacidades de las personas para llevar a cabo el tipo de vida que tienen razones para valorar (Sen, 1999). Para el PNUD, “el desarrollo humano consiste en la ampliación de las capacidades de todas las personas para que puedan libremente elegir lo que desean ser y hacer, de manera que puedan gozar de una vida prolongada, saludable y creativa, perseguir objetivos que consideren valiosos y participar activamente en el desarrollo sostenible y equitativo del planeta que comparten" (2010: 2). El desarrollo, por tanto, es la libertad, pero una libertad real, posible y garantizada. Es la garantía del pleno ejercicio de los derechos fundamentales, pero también de medios materiales, ingresos y riqueza, oportunidades, acceso a servicios de educación o salud, es poder expresarse en democracia y tener seguridad, 
poder vivir en un medio ambiente sano alrededor, y es también la confianza y la autoestima en el propio proyecto vital (Ministerio de Asuntos Exteriores y Cooperación, 2013: 27).

Si el desarrollo es libertad, la pobreza es falta de libertad, es no poder llevar a cabo la vida que se desea, que racionalmente se valora. Es un concepto de pobreza mucho más complejo que las mediciones de pobreza de ingreso del Banco Mundial, e incluso del Índice de Desarrollo Humano del PNUD, o de las mediciones de cualquier otro organismo internacional. Esta es una visión más profunda, que trasciende también la Agenda 2030. El objetivo es mucho más ambicioso, es antropológico, ético y vital, y esto es lo que debe ser logrado para todos, sin dejar a nadie atrás.

Esta libertad se concreta en el enfoque de las capacidades, lo que las personas son capaces de hacer y ser, teniendo en cuenta su propia diversidad y naturaleza (Pedrajas, 2007). Por eso la ética del desarrollo humano debe ser todavía más argumentada y explorada en las posibilidades antropológicas del enfoque de las capacidades tal y como propone Jesús Conill (2004). El fundamento de las capacidades no puede estar en un liberalismo basado en el velo de la ignorancia de Rawls, las capacidades las tienen, o no las tienen, las personas de carne y hueso, hombres y mujeres, niños, jóvenes, adultos y mayores, que pertenecen a una comunidad concreta, que son de una raza o etnia, que tienen o no tienen alguna discapacidad, que tienen una propensión a enfermar, que tienen unas habilidades, una inteligencia, unas aspiraciones y proyectos vitales limitados por unas capacidades, que es lo que pueden hacer y ser, y que hay que ampliar al máximo para que puedan ponerlas al servicio de la vida que desean llevar, para que puedan realizar su proyecto vital. Esto y no otra cosa, es el desarrollo humano, y la solidaridad con los más desfavorecidos, con los que no han tenido suerte en la lotería natural, es de justicia social (Rawls, 1995; 1996). Por eso es preciso es aterrizar las capacidades en la naturaleza humana, concreta y real de las personas desfavorecidas, y empoderar a esas personas, ampliar sus capacidades. Los programas de desarrollo del PNUD, desde hace veinticinco años, han basado toda su fundamentación en esta ampliación de capacidades, en un capacity building, pero también de las instituciones locales, gobiernos regionales y nacionales. Es preciso empoderar a las personas en sus posibilidades para que tengan la oportunidad de hacer con su vida lo que realmente desean y valoran, y es preciso también construir capacidades en las instituciones responsables de mejorar las políticas públicas para que realmente puedan lograrlo y mejorar la vida de sus ciudadanos (Pedrajas, 2007; PNUD, 1990-2017).

Finalmente, el tercer pilar es el modelo de persona moral que propone Amartya Sen como fundamento del modelo de desarrollo humano. Un modelo basado en "agencia" y "bienestar", pero que también puede y debe 
ser mejor fundamentado de manera coherente con una ética del desarrollo humano. Para nosotros, debe estar basado en un modelo de autonomía (que supere la mera "agencia”). El ser humano es valioso en sí mismo y dueño de su propia vida y desarrollo, y eso es lo que hay que empoderar, se deben ampliar las capacidades de tomar decisiones personales, sociales y políticas. Como parte de la vida política y de un modelo de desarrollo, el concepto de autonomía es fundamental, basado en la dignidad humana y en los derechos humanos (Pedrajas, 2005; 2007).

Por tanto, desarrollo como libertad, fortalecimiento y expansión de capacidades de los más desfavorecidos y empoderamiento y autonomía moral son los tres pilares que fundamentan un modelo de desarrollo que debe inspirar el desarrollo internacional, y en concreto ahora, de la Agenda 2030 y los ODS.

\section{LA AGENDA 2030 PARA EL DESARROLlo SOSTENIBLE}

La agenda 2030 es una agenda centrada en las personas, con un modelo de desarrollo humano y sostenible, que cuida el planeta y promueve la prosperidad y la paz, con un compromiso ético poderoso: Leaving No One Behind, sin dejar a nadie atrás. Es una agenda que impulsa los principios y valores de la Carta de Naciones Unidas y que promueve el bien común.

Agenda 2030, punto 4: "Al emprender juntos este gran viaje, prometemos que nadie se quedará atrás. Reconocemos que la dignidad de la persona bumana es fundamental, por lo que deseamos ver cumplidos los Objetivos y las metas para todas las naciones y los pueblos y para todos los sectores de la sociedad, y nos esforzaremos por llegar primero a los más rezagados" (Naciones Unidas, 2015a).

El punto de partida es la culminación del periodo 2000-2015 definido por los Objetivos de Desarrollo del Milenio (ODM) y la propia revisión de otros procesos y cumbres internacionales de desarrollo, fundamentalmente Río+20. La definición de la nueva agenda generó un proceso de negociación sin precedentes, inclusivo y participativo, que ha construido esta agenda "bottom-up" que le ha dado una legitimidad ética, social y política de la que carecían otras agendas de desarrollo anteriores ${ }^{1}$.

La nueva agenda no solo destaca por el proceso de construcción de la misma, también por sus contenidos innovadores y ambiciosos para afrontar los retos del mundo global en la próxima década. Podemos definir tres

1 Del proceso participativo de construcción de la agenda destacan los siguientes informes: Panel de Alto Nivel de Personas Eminentes, 2013; PNUD, 2013a; SDSN, 2013; UN Global Compact, 2013; Grupo de Desarrollo de las Naciones Unidas, 2013; Grupo Abierto para los ODS, 2014; Secretariado General de Naciones Unidas, 2014. 
características principales (Secretaría General de Cooperación Internacional para el Desarrollo, 2015; Pedrajas, 2014):

- Es una agenda universal: que compromete a todos los países. No es una agenda de países ricos para que la cumplan los países pobres, es la agenda de todos. La agenda del desarrollo sostenible, que solo es posible si todos los países se comprometen a aplicarla en sus territorios, a descentralizarla a niveles regionales y locales. Cada uno con sus propias características y responsabilidades diferenciadas, pero todos los países del mundo son ahora países en desarrollo (Banco Mundial, 2016).

- Una agenda transformadora: que va a las causas, que no se centra solo en las consecuencias o en paliar los efectos de la pobreza extrema y prestar servicios básicos. Va también al origen, a la falta de capacidades en las instituciones, a la falta de buena gobernanza democrática y de derechos o acceso a la justicia. Por esto es una agenda que apunta también a procesos tan importantes como los sistemas de producción y consumo, o a las ciudades, que deben ser sostenibles. Una agenda que equilibra las tres dimensiones del desarrollo: económico, social y ambiental, de manera que se han integrado objetivos tan novedosos ( $\mathrm{y}$ tan ausentes en la anterior del milenio) como el cambio climático, la protección de la biodiversidad o de los ecosistemas.

- Una agenda inclusiva: no deja a nadie atrás, es una agenda centrada en la dignidad de las personas que se compromete en luchar e incluir a todas y cada una, sin distinción, sin exclusión por motivos de sexo, edad, discapacidad, origen étnico, geográfico o ninguna otra causa o justificación. En la Declaración política de la Agenda 2030 se negoció y se logró que se incluyeran a todas las personas y sectores de la sociedad, algo que parece obvio, pero no es tan evidente cuando se negocia con todos los países de Naciones Unidas (Pedrajas, 2015). El fundamento de los derechos humanos es central en este punto y debe defenderse.

Nos encontramos, por tanto, con una nueva agenda universal de desarrollo, con responsabilidades comunes pero diferenciadas para los países, basada en una apropiación democrática, y con un objetivo claro: la erradicación de la pobreza y la promoción del desarrollo sostenible para todas las personas.

Es importante en este punto adentrarnos también en los valores del desarrollo. En la Agenda del Milenio se afirmaban los valores y principios 
y se describían explícitamente en el punto seis de la Declaración del Milenio. Los valores que inspiraban la agenda de desarrollo y las relaciones internacionales para el siglo XXI: la libertad, la igualdad, la solidaridad, la tolerancia, el respeto de la naturaleza y la responsabilidad común (Naciones Unidas, 2000).

En la Agenda 2030 el debate ha sido más político, económico, técnico y, solo al final, cuando se negoció el preámbulo y la declaración política, salieron a la luz los fundamentos, principios y valores de la nueva agenda. Sin aparecer como tal los valores del desarrollo, se reafirma la Declaración del Milenio, y se centra más en la universalidad de la nueva agenda para todos los países y al equilibrio entre las tres dimensiones del desarrollo sostenible.

En este sentido, el preámbulo de la nueva Agenda 2030 es muy inspirador, enmarcando los cinco grandes principios que deben guiar el trabajo concretado posteriormente por los 17 ODS: Personas, Planeta, Prosperidad, Pary Alianzas.

La Declaración política va más allá del preámbulo, tiene una visión ética global, que no entra en un debate profundo sobre los fundamentos ni en cuestiones antropológicas (sería un debate muy complicado en el seno de Naciones Unidas) pero el acuerdo son los principios comunes que guían el trabajo de todos los países en su conjunto, y eso tiene un gran valor ético. Son los mínimos éticos que ponen a todos de acuerdo, en un mundo muy diverso y complejo, son los derechos humanos y la dignidad humana lo que sustenta el trabajo común por la justicia, el desarrollo y la paz.

En la Agenda 2030 encontramos una visión de futuro, en el punto 7 se afirma: "aspiramos a un mundo sin pobreza, hambre, enfermedades ni privaciones, donde todas las formas de vida pueden prosperar; un mundo sin temor ni violencia".

Y sigue el punto 8: "aspiramos a un mundo en el que sea universal el respeto de los derechos humanos y la dignidad de todas las personas, el estado de derecho, la justicia, la igualdad y la no discriminación. (...)

Nuestros principios y compromisos comunes, afirma los principios de la Carta de Naciones Unidas, los fundamentos de la Declaración de Derecho Humanos, la Declaración del Milenio..." (Naciones Unidas, 2015a).

Por tanto, esta agenda tiene una visión ética, construida sobre los principios y valores de Naciones Unidas, en los que podría profundizarse en la línea de la ética del desarrollo humano, y que pueden ser reforzados a lo largo de su puesta en práctica. Nuestro objetivo en ese artículo está en esta línea, en tratar de reforzar los argumentos éticos, pero no tratando de abordar toda la agenda, si no centrándonos en la lucha contra la pobreza extrema y la vulnerabilidad, en la última milla. 
4. LA CENTRALIDAD DE LA PERSONA: NO DEJAR A NADIE ATRÁS. LA ÚLTIMA MILLA DEL DESARROLLO

Durante la construcción de la nueva agenda, uno de los debates que pasó más desapercibido era, sin embargo, sobre un tema fundamental, fue la discusión de si debería ser una agenda "people-centred" o debía equilibrar personas, planeta y prosperidad en el mismo nivel. Tanto la posición española en su momento (Secretaría General de Cooperación Internacional para el Desarrollo, 2015), como la del PNUD, apostaban por mantener la centralidad de la persona, y en un buen equilibrio con la importancia de la protección del planeta, y podemos afirmar que así se logró. De esta manera el reconocimiento de la dignidad de la persona y los derechos humanos son fundamentales en la nueva agenda, pero ciertamente en un marco muy bien equilibrado con las tres dimensiones del desarrollo, donde el planeta y los límites planetarios debían estar incorporados en un fuerte impulso de la sostenibilidad que tiene en cuenta nuestra casa común (Francisco, 2015) y las generaciones futuras. La generación de prosperidad es el tercer motor del desarrollo, que debe estar al servicio del a persona y de su bienestar y libertad.

Tanto el Preámbulo, como la Declaración como la mayoría de los Objetivos (al menos 8 de los 17 son de claro contenido social) ponen al ser humano en el centro. Reafirmado por un compromiso ético de primer orden en esta nueva agenda, el desarrollo sostenible debe llegar a todos los seres humanos, sin exclusión ninguna, esta agenda no debe dejar a nadie atrás.

Este compromiso ético, uno de los más fuertes de la nueva agenda de desarrollo, ha impulsado que los primeros años de su puesta en práctica ya hayan estado centrados en el análisis de quienes son los que se quedan atrás, quienes son los más rezagados, los excluidos y los marginados, y sobre todo, porque se están quedando atrás para poder aplicar las políticas públicas necesarias para llegar a ellos, para que el desarrollo sea verdaderamente inclusivo (United Nations, 2016).

El PNUD está trabajando este enfoque aterrizándolo todavía más, conocido como la Última Milla e impulsado a través del informe: Getting to the Last Mile in Least Developed Countries (Pedrajas \& Choritz, 2016), presentado en la Conferencia de Revisión del Plan de Acción para los Países Menos Adelantados celebrada en Antalya en Mayo de 2016. En esta obra por última milla se refiere a los más pobres de los pobres, pero también a todas las personas, lugares incluso las todas aquellas pequeñas empresas que están insuficientemente atendidas y excluidas, donde las necesidades de desarrollo son mayores y donde los recursos son más escasos. 
Como se establecía en la introducción de este artículo, los datos nos dicen que la gente en la mayoría de los países ha ido mejorando en los niveles de desarrollo humano. Los avances en la tecnología, la educación y los ingresos prometen cada vez una vida más plena, con mejor calidad de vida y más segura. Aunque también es cierto que se da en entorno de inestabilidad e incertidumbre y, sobre todo, hay todavía millones de personas no se están beneficiando de los frutos del desarrollo, especialmente los más pobres y los desfavorecidos a causa de su sexo, edad, discapacidad, origen étnico o geográfico. Esta es la última milla. Pero no es una distancia física, ni un tramo que recorrer, es una última milla moral. Son 766 millones de personas en 2016 (Banco Mundial, 2017; UNICEF \& World Bank Group, 2016. Y consultar también Pedrajas \& Choritz, 2016), que siguen sobreviviendo en condiciones de pobreza extrema y marginalidad, en los países más pobres del mundo "el segmento más pobre y débil de la comunidad internacional" (Naciones Unidas, 2011), pero también en los suburbios de las ciudades y en las regiones industrializadas y países avanzados.

La última milla es, además, la más difícil de recorrer. Podemos entender el fenómeno que se produce de persistencia de la pobreza generación tras generación desde dos puntos de vista, uno estructural y otro dinámico.

\subsection{Análisis estructural de la última milla}

Como causas estructurales de la última milla, encontramos la combinación de pobreza extrema reforzada por la desigualdad y la exclusión social. Por tanto, no sirven solo políticas de crecimiento económico inclusivo, o de infraestructuras y transferencias de recursos para cubrir esta última milla. Haciendo lo mismo que hasta ahora, se seguirá reduciendo la pobreza extrema, pero no se erradicará, porque nunca se cubrirá esa última milla. Es preciso abordar las causas profundas de las desigualdades (horizontales) y la exclusión social desde un enfoque de derechos humanos. Todo ello nos lleva a un análisis estructural basado en enfoque multidimensional de pobreza, desigualdad y exclusión.

Esto viene explicado también por el Banco Mundial que señala que según la pobreza extrema se reduce, el crecimiento por sí solo tiende a sacar a un menor número de personas de la pobreza. Esto se debe a que algunos pobres permanecen fuera de la corriente que los beneficios que generan estos patrones de crecimiento; porque viven en áreas que están más allá del alcance de la infraestructura existente, de los servicios sociales, o de las finanzas; Pueden ser miembros de un grupo minoritario y desfavorecido, ser particularmente vulnerables a los efectos de un desastre o una crisis y carecen de mecanismos adecuados para afrontarlos, o viven en zonas remotas donde es poco probable que tenga fuertes vínculos con la 
creación de empleo del crecimiento económico. Por lo tanto, el crecimiento tiene que ser más inclusivo y relevante para la vida de los hogares pobres y las comunidades, y los programas y las estrategias deben abordar las barreras estructurales para su inclusión en los beneficios del crecimiento (Chandy, Kato \& Kharas, 2015).

Pero, sobre todo, no es una cuestión solo de crecimiento económico. En la última milla, la pobreza, la vulnerabilidad, las desigualdades y la exclusión a menudo se refuerzan mutuamente.

Por ello, la aproximación debe ser multidimensional. La Agenda 2030, en su preámbulo, subraya que: “...la erradicación de la pobreza en todas sus formas y dimensiones, como la pobreza extrema, es el mayor desafío y una condición indispensable para el desarrollo sostenible”. La pobreza extrema es una manifestación de las exclusiones y desigualdades persistentes, lo que resulta en las personas que se quedan atrás. En este sentido, el concepto de pobreza multidimensional nos ayuda a comprender estas interrelaciones y también las desigualdades profundas y exclusiones que pueden hacer que la pobreza sea tan persistente e incluso perdure entre las generaciones (Alkire \& Santos, 2010).

Y como refuerzo al enfoque de pobreza multidimensional, para hacer frente a las condiciones estructurales que dejan a las personas atrás, tenemos que entender los diferentes tipos de desigualdades a los que se enfrentan. La desigualdad no es solo un problema en si mismo, tiene efectos negativos sobre la capacidad de crecimiento para reducir la pobreza. Es decir, la desigualdad alta refuerza la persistencia de la pobreza extrema, ya que fomenta una tasa inicial mayor de pobreza que, a su vez, genera más pobreza.

Algunos autores argumentan que un mayor nivel de pobreza inicial tiende generar más pobreza. Este hallazgo puede explicarse por la gente pobre, que experimentan condiciones únicas que les atrapan en la pobreza convirtiéndose en aún más pobres. Muchas de estas condiciones —tales como la falta de propiedad del capital (humano, físico y/o financiero)son el resultado de la desigual distribución inicial.

Por tanto, no abordar la desigualdad puede atrapar a las personas en la pobreza persistente. Esta es una observación hecha por Alexis de Tocqueville en el siglo XIX: en la base de la pirámide, se encuentra el principio de la desigualdad, que se ha actualizado por medio del concepto de desigualdades horizontales de Francis Stewart (2009; Stewart \& Langer, 2007).

Las desigualdades horizontales son las que existen entre los grupos con características específicas que sus miembros y otros reconocen como aspectos importantes de su identidad (PNUD, 2013b). Estos grupos pueden ser definidos por la cultura, el género, el origen étnico, la religión, la 
raza, la ubicación geográfica, o edad, entre otras características. Las desigualdades horizontales son el resultado de la discriminación y la exclusión sistémica que normalmente se derivan de los estereotipos y los prejuicios. Estas desigualdades se relacionan con la desigualdad de oportunidades, y tienen experiencia en relación con los derechos y el acceso a los servicios sociales, educación, salud, nutrición, vivienda, bienes, justicia, empleo y recursos económicos. Es decir, vuelve a ser necesario un enfoque multidimensional con el que abordar la desigualdad.

Un enfoque también reforzado por la exclusión en la última milla y de la falta de respeto a los derechos humanos. Por exclusión entendemos un proceso en el cual los miembros de un grupo minoritario son considerados de manera diferente (menos favorable) o se les niega el acceso completo a los derechos, oportunidades y recursos que normalmente están disponibles a los miembros de un grupo diferente (la mayoría) en similares circunstancias. Afecta, por ejemplo, pobres urbanos y rurales; las poblaciones indígenas; minorías étnicas o sexuales; gente con discapacidades; personas que viven con el VIH; inmigrantes; refugiados, desplazados internos; mujer; y la juventud.

Por tanto, desde un análisis ético de la nueva agenda, el compromiso de no dejar a nadie atrás se refuerza con un enfoque multidimensional para abordar la pobreza extrema, la desigualdad y la exclusión en la última milla con el objetivo de empoderar a las personas y fortalecer sus capacidades para poder iniciar una vida de desarrollo humano, empezar a vislumbrar el desarrollo como libertad. Las políticas de desarrollo deben abordar desde las desigualdades y la exclusión los desafíos de la pobreza extrema. Esto podría tomar la forma, por ejemplo, de las políticas fiscales que favorezcan a los pobres; programas de protección social que proporcionan fuertes redes de seguridad social para las personas más excluidas; el acceso universal a los servicios básicos de calidad; y el acceso equitativo a los recursos naturales y productivos. Se podría tomar la forma de ampliar la participación en la vida pública y política. Y pone de relieve la importancia de la lucha contra la discriminación —es decir, los prejuicios, los estereotipos y otras normas culturales que reproducen las desigualdades- mediante la aplicación de la legislación y las reformas antidiscriminatoria destinada a aumentar el acceso a los grupos desfavorecidos a mejores condiciones de vida (Pedrajas \& Choritz, 2016: 14).

\subsection{La vulnerabilidad}

Frente al enfoque más estructural definido por la pobreza-desigualdad y exclusión, para afrontar la vulnerabilidad nos referimos a un enfoque dinámico. 
Es necesario ver la última milla a través de un enfoque dinámico, no solamente se trata de sacar a la gente de la pobreza, se trata de mantenerlos fuera de la pobreza. Los logros tienen que ser estables, tienen que ser duraderos a largo plazo y es preciso trabajar en estos entornos teniendo en cuenta que los shocks o las crisis que afectan a las personas vulnerables pueden hacerles perder todo. Nos referimos sobre todo a los riesgos que vienen de los desastres naturales, a los efectos del cambio climático, los brotes de enfermedades, los conflictos o crisis políticas y económicas.

Las políticas que sacan a la gente de la pobreza son distintas a las que les mantienen fuera de la pobreza, por eso deben estar bien informadas de los riesgos y ser implementadas teniendo en cuenta la necesaria capacidad de recuperación frente a los shocks. La protección social es fundamental en este contexto. Las familias, las comunidades deben tener la infraestructura necesaria y ser capaces de adaptarse y resistir los shocks, cualesquiera que sean, sin tener que vender o perder lo poco que poseen y caer de nuevo en pobreza extrema.

Por esta razón, en el ODS 1 se incluyen dos metas destinadas a aumentar la resiliencia de las personas vulnerables:

1.2. Implementar sistemas nacionales apropiadas de protección social y medidas para todos, incluidos pisos, y en 2030 lograr una cobertura sustancial de los pobres y los vulnerables.

1.5 Para el año 2030, aumentar la resiliencia de los pobres y los que están en situación de vulnerabilidad y reducir su exposición y la vulnerabilidad a los fenómenos extremos relacionados con el clima y otros impactos económicos, sociales y ambientales y desastres.

Las políticas que pueden dar soluciones concretas y que pueden ayudar a construir resiliencia y afrontar las vulnerabilidades a nivel nacional y subnacional incluyen el fortalecimiento de la protección social, la promoción del pleno empleo y el trabajo decente, la construcción de instituciones capaces y fuertes, inversión en la cohesión social, fortalecimiento de capacidades para prepararse y recuperarse de la crisis y un enfoque en la mitigación y adaptación a los riesgos, incluido el cambio climático (Pedrajas \& Choritz, 2016; Pedrajas, 2011-2012).

Por tanto, un enfoque multidimensional que promueva el desarrollo humano es fundamental para hacer frente a los desafíos dela última milla. Haciendo lo mismo que se ha hecho hasta ahora, se reducirá la pobreza, pero no se erradicará. Y tenemos el acuerdo político de más alto nivel en el seno de Naciones Unidas, sabemos que existen los medios económicos y los recursos para poder llevarlo a cabo, falta realmente una voluntad de poner en marcha las políticas públicas necesarias que permitan llegar a los 
casi mil millones de personas que viven en pobreza extrema y vulnerabilidad. Falta llegar a ellos, saber quiénes son y donde están, por qué están en esa situación generación tras generación. Y una vez allí, fortalecer sus capacidades, empoderar su autonomía y empezar a darles las oportunidades para poder subir el primer eslabón del desarrollo, y no bajar de él, es el eslabón más difícil, pero que algún día se convertirá en la clave que permitió iniciar una vida de desarrollo como libertad.

\section{REFERENCIAS}

-Alkire, S. \& Santos, M. E. (2010). Acute Multidimensional Poverty: A New Index for Developing Countries (Working Paper, n. 38). Oxford: Oxford University Poverty Initiative.

-Banco Mundial (2016). Informe Desarrollo Mundial 2016. Washington DC: Banco Mundial.

-Banco Mundial (2017). PovcalNet [base de datos en línea]. Recuperado de http:/ /iresearch.worldbank.org/PovcalNet/povDuplicateWB.aspx

-Chandy, L., Kato, H. \& Kharas H. (2015). The Last Mile in Ending Extreme Poverty. Washington DC: Brookings Institution Press.

-Collier, P. (2007). The Bottom Billion: why the poorest countries are failing and what can be done about it. Oxford: Oxford University Press.

-Conill, J. (2004). Horizontes de economía ética. Madrid: Tecnos.

-Cortina, A. (1993). Ética aplicada y democracia radical. Madrid: Tecnos.

-Cortina, A. (1997). Ciudadanos del mundo. Madrid: Alianza.

-Cortina, A. (2001). Alianza y contrato. Madrid: Trotta.

-Cortina, A. (2002). Por una ética del consumo. Madrid: Taurus.

-Cortina, A. (2017). Aporofobia: el rechazo al pobre. Barcelona: Paidós.

-Domingo, A. (1997). Ética y voluntariado: una solidaridad sin fronteras. Madrid: PPC. -Francisco (2015). Carta encíclica Laudato si'. Sobre el cuidado de la Casa Común. Recuperado de http://w2.vatican.va/content/francesco/es/encyclicals/documents/papa-francesco_20150524_enciclica-laudato-si.html

-Goulet, D. (1985). The Cruel Choice: A New Concept in the Theory of Development. New York: University Press of America.

-Goulet, D. (1999). Ética del desarrollo. Madrid: IEPALA.

-Grupo abierto para los ODS (2014). Informe Final Objetivos de Desarrollo Sostenible. Recuperado de https:/ / sustainabledevelopment.un.org/focussdgs.html

-Grupo de Desarrollo de las Naciones Unidas (2013). Post2015: Inicia la conversación global. Recuperado de http://www.undp.org/content/undp/es/home/librarypage/mdg/global-conversation-begins

-Martínez, E. (2000). Ética para el desarrollo de los pueblos. Madrid: Trotta.

-Martínez-Solimán, M. \& Conceiçāo, P. (2017). La ONU del desarrollo, el clima y la paz. Politica Exterior, (177).

-Ministerio de Asuntos Exteriores y Cooperación (2013). Plan Director de la Cooperación Española 2013-2016. Madrid. 
-Naciones Unidas (1945). Carta de las Naciones Unidas. San Francisco. Recuperado de http://www.un.org/es/charter-united-nations/index.html

-Naciones Unidas (2000). Resolución aprobada por la Asamblea General A/RES/55/2*. Declaración del Milenio. Recuperada de http://www.un.org/spanish/milenio/ares552.pdf

-Naciones Unidas (2005-2015). Informe de los Objetivos de Desarrollo del Milenio. Informes anuales desde 2005 hasta 2015, Nueva York. Recuperados de http://www.un.org/es/millenniumgoals/reports.shtml

-Naciones Unidas (2011). Plan de Acción de Estambul para los Países Menos Adelantados. Resolución A/CONF.219/3. Recuperado dehttp://unohrlls.org/UserFiles/File/IPoA.pdf

-Naciones Unidas (2015a). Transformar nuestro mundo: la Agenda 2030 para el Desarrollo Sostenible. Resolución de la Asamblea General A/71/1, aprobada el 25 de septiembre de $2015 . \quad$ Recuperada de http://www.un.org/ga/search/view_doc.asp?symbol=A/RES/70/1\&referer $=/$ english $/ \&$ Lang $=\mathrm{S}$

-Naciones Unidas (2015b). Objetivos de Desarrollo del Milenio. Informe de 2015. Recuperado de http:/ / www.un.org/es/millenniumgoals/pdf/2015/mdg-report2015_spanish.pdf

-Naciones Unidas (2015c). Agenda de Acción de Addis Abeba. Resolución A/RES/69/313, aprobada por la Asamblea General el 27 de julio de 2015. Recuperado de http://www.un.org/en/ga/search/view_doc.asp?sym$\mathrm{bol}=\mathrm{A} / \mathrm{RES} / 69 / 313$

-Naciones Unidas (2016a). Report of the Interagency and Expert Group on Sustainable Development Goal Indicators. Aprobado por ECOSOC el 7 de Junio de 2017, Nueva York. Recuperado de https://unstats.un.org/sdgs/files/meetings/iaeg-sdgs-meeting-05/2017-2-IAEG-SDGs-E.pdf

-Naciones Unidas (2016b). Informe de los Objetivos de Desarrollo Sostenible 2016. Nueva York. Recuperado de https://unstats.un.org/sdgs/report $/ 2016 /$ the $\% 20$ sustainable $\% 20$ development $\% 20$ goals $\% 20$ report $\% 202016 \_s p a n i s h . p d f$

-Panel de Alto Nivel de Personas Eminentes (2013). Informe Una nueva alianza mundial: erradicar la pobreza y transformar las economias a través del desarrollo sostenible. Recuperado de http://www.un.org/sg/management/pdf/HLP_P2015_Report.pdf.

-Pedrajas, M. (2005). El desarrollo bumano en la economia ética de Amartya Sen. Tesis Doctoral. Valencia: Universitat de Valencia.

-Pedrajas, M. (2007). Ética, desarrollo y democracia a partir del enfoque de las capacidades de A. Sen. Diálogo Filosófico, (69), 401-418.

-Pedrajas, M. (2011-2012). El impacto de la crisis: pautas para una agenda de protección social. Economía Exterior, (59), 109-118.

-Pedrajas, M. (2014). Prioridades de la posición española para la Agenda post2015. Revista ICE “Globalización y Desarrollo”, (880), 127-140.

-Pedrajas, M. (2015). Entrevista: "No esperaba que fuera tan difícil negociar sobre Derechos Humanos". Planeta Futuro, 23 de septiembre. Recuperada de 
https://elpais.com/elpais/2015/09/11/planeta_futuro/1441991073_286184.html

-Pedrajas, M. \& Choritz, S. (2016). Getting to the Last Mile in Least Developed Countries. New York: PNUD y UNCDF.

-PNUD (1990-2017). Informe de Desarrollo Humano, todos los años 1990-2017, Nueva York. http://hdr.undp.org/

-PNUD (2010). Informe de Desarrollo Humano 2010, Nueva York. Recuperado de http://hdr.undp.org/sites/default/files/hdr_2010_es_complete_reprint.pdf

-PNUD (2013a). Consultas temáticas y nacionales UNDG, Un millón de voces: el mundo que queremos. Recuperado de http://www.undp.org/content/undp/es/home/librarypage/mdg/a-million-voices--the-world-wewant/

-PNUD (2013b). Humanity Divided: confronting inequality in developing countries, Nueva York. Recuperado de http://www.undp.org/content/dam/undp/library/Poverty $\% 20$ Reduction/Inclusive $\% 20$ development/Humanity $\% 20$ Divided/HumanityDivided_Full-Report.pdf

-PNUD (2017). Informe de Desarrollo Humano 2016: Desarrollo Humano para todas las Personas. Nueva York. Recuperado de http://hdr.undp.org/sites/default/files/hdr_2016_report_spanish_web.pdf

-Rawls, J. (1995). Teoría de la justicia. Madrid: FCE.

-Rawls, J. (1996). El liberalismo politico. Barcelona: Crítica.

-SDSN (2013). Una agenda de acción para el desarrollo sostenible. Recuperado de http://unsdsn.org/wp-content/uploads/2014/02/Una-Agenda-de-Acción-para-el-Desarrollo-Sostenible.pdf

-Secretaría General de Cooperación Internacional para el Desarrollo (2015). Compromiso Universal por un Desarrollo Humano y Sostenible. Posición española para la Agenda Post2015. Documento de Trabajo n. 6. Madrid: Cooperación Española.

-Secretariado General de Naciones Unidas (2014). Informe de Síntesis: "El camino a la dignidad para 2030: acabar con la pobreza y transformar vidas protegiendo el planeta”. Recuperado de http://www.un.org/ga/search/view_doc.asp?symbol=A/69/700\&Lang $=\mathrm{S}$

-Sen, A. (1989). Sobre ética y economía. Madrid: Alianza.

-Sen, A. (1999). Development as Freedom. New York: A. Knopf.

-Stewart, F. (2009). Horizontal inequalities as a cause of conflict. Bradford Development Lecture.

-Stewart, F. \& Langer, A. (2007). Horizontal inequalities: explaining persistence and change. CRISE Working Paper, 39. University of Oxford.

-UN Global Compact (2013). Corporate Sustainability and UN post2015 Agenda, junio 2013. Recuperado de https://www.unglobalcompact.org/docs/news_events/9.1_news_archives/2013_06_18/UNGC_Post2015_Report.pdf

-UNICEF \& World Bank Group (2016). Ending Extreme Poverty: A Focus on Children. New York: UNICEF \& Wold Bank Group. 
-United Nations Framework Convention on Climate Change (2015). Resolution to Adoption of Paris Agreement, 12 December. Recuperado de https://unfccc.int/resource/docs/2015/cop21/eng/109r01.pdf

-United Nations (2016). Global Sustainable Development Report 2016: Leaving No One Bebind. New York: Department of Economic and Social Affairs.

Sumario: 1. Introducción: ética global para un mundo global; 2. Los principios de la ética del desarrollo humano; 3. La Agenda 2030 para el Desarrollo Sostenible; 4. La centralidad de la persona: No dejar a nadie atrás. La última milla del desarrollo; 4.1. Análisis estructural de la última milla; 4.2. La vulnerabilidad; Referencias. 